\title{
On the port-Hamiltonian representation of systems described by partial differential equations
}

\author{
M. Schöberl* A. Siuka** \\ * Johannes Kepler University Linz, Institute of Automatic Control and \\ Control Systems Technology, Austria \\ (e-mail: markus.schoeberl@jku.at) \\ ** Johannes Kepler University Linz, Austria \\ (e-mail: a.siuka@liwest.at)
}

\begin{abstract}
We consider infinite dimensional port-Hamiltonian systems. Based on a power balance relation we introduce the port-Hamiltonian system representation where we pay attention to two different scenarios, namely the non-differential operator case and the differential operator case regarding the structural mapping, the dissipation mapping and the in/output mapping. In contrast to the well-known representation on the basis of the underlying StokesDirac structure our approach is not necessarily based on using energy-variables which leads to a different port-Hamiltonian representation of the analyzed partial differential equations.

NOTICE: this is the authors version of a work that was accepted for publication in ifacpapersonline.net. Changes resulting from the publishing process, such as peer review, editing, corrections, structural formatting, and other quality control mechanisms may not be reflected in this document. Changes may have been made to this work since it was submitted for publication. A definitive version was subsequently published in ifac-papersonline.net, DOI 10.3182/201208293-IT-4022.00001
\end{abstract}

Keywords: Differential geometric methods, Hamiltonian Systems, Partial differential equations, System theory

\section{INTRODUCTION}

Modeling of physical systems described by partial differ' ential equations (pdes) in a port-Hamiltonian framework has been treated extensively over the last years. However, there are still many open issues and there exists no unique representation. An important contribution in this field was the introduction of Stokes-Dirac structures which allows to analyze field theories in a port-Hamiltonian framework and to exploit this system representation for the controller design, see e.g. van der Schaft and Maschke (2002); Macchelli et al. (2004a,b); Macchelli and Melchiorri (2004); Maschke and van der Schaft (2005). Roughly speaking, the key property of the Stokes-Dirac structure is to represent the power balance relation of physical systems in an formal way. This is achieved by combining so-called flows and efforts in the domain and on the boundary of a system. Using this approach the proper choice of energy variables is crucial and the Hamiltonian is also a functional depending on energy variables.

A different kind of a port-Hamiltonian representation, not focusing on energy variables, but also based on a power conservation law, was proposed in Schlacher (2008); Schöberl et al. (2008). The proposed Hamiltonian framework can be seen as an extension of Olver (1986) by incorporating dissipation and boundary ports. This formulation has been exploited for control issues in Schöberl and Siuka (2011) and Siuka et al. (2011).

In this contribution we will present an enhancement of Schlacher (2008); Schöberl et al. (2008) in such a way that beside the input map as in Schöberl et al. (2008) also the structural mapping $\mathcal{J}$ and the dissipation mapping $\mathcal{R}$ may involve differential operators.

In the approach based on Stokes-Dirac structures it is quite natural that these mappings involve differential operators, however due to the choice of energy variables the Hamiltonian density does not depend on derivative variables, in contrast to the approach presented in this paper. For instance in mechanics, the choice of energy variables suggests to use the strain $\epsilon$ as the dependent coordinate, whereas also the displacement $u$ can be used. This latter choice leads to derivative coordinates when the potential energy has to be stated, since the energy is a function of the strain and $\epsilon=\partial_{X} u$ is met in the case of a one-dimensional domain. This has severe consequences in the application of the variational derivative applied to the Hamiltonian density, leading to a different portHamiltonian representation, and has also an impact on the computation of structural invariants, see also Schöberl and Siuka (2011).

This paper is organized as follows. In Section 2 some notation is presented and the geometric objects that play 
a fundamental role in the paper are introduced. The third section is dealing with the representation of portHamiltonian systems described by pdes, where we focus on two cases: the non-differential operator case and the differential operator case. Two specific applications, the vibrating string and a simple model of magnetohydrodynamics are analyzed in the fourth section to demonstrate how the introduced differential operators have an impact on the power balance in practice. The paper closes with some concluding remarks.

\section{NOTATION AND PRELIMINARIES}

In this paper we will apply differential geometric methods and we will use a notation that is similar to the one in Giachetta et al. (1997). To keep the formulas short and readable we will use tensor notation and especially Einsteins convention on sums. We use the standard symbol $\otimes$ for the tensor product, $\wedge$ denotes the exterior product (wedge product), $\mathrm{d}$ is the exterior derivative, $\rfloor$ the natural contraction between tensor fields. By $\partial_{\alpha}^{B}$ are meant the partial derivatives with respect to coordinates with the indices ${ }_{B}^{\alpha}$. Furthermore $C^{\infty}(\cdot)$ denotes the set of the smooth functions on the corresponding manifold. Moreover we will not indicate the range of the used indices when they are clear from the context. Additionally pull back bundles are not indicated to avoid exaggerated notation.

In the forthcoming we will consider bundle structures in order to be able to separate dependent and independent coordinates. Let us consider the bundle $\mathcal{X} \rightarrow \mathcal{D},\left(X^{A}, x^{\alpha}\right) \rightarrow$ $\left(X^{A}\right)$ where $x$ are the dependent and $X$ the independent coordinates. The first jet manifold $\mathcal{J}^{1}(\mathcal{X})$ can be introduced possessing the coordinates $\left(X^{A}, x^{\alpha}, x_{A}^{\alpha}\right)$, where the capital Latin indices $A, B$ are used for the base manifold $\mathcal{D}$ (independent coordinates) and $x_{A}^{\alpha}$ denote derivative coordinates of first order (derivatives of the dependent coordinates with respect to the independent ones). The jet structure also induces the so-called total derivative

$$
d_{A}=\partial_{A}+x_{A}^{\alpha} \partial_{\alpha}+x_{A B}^{\alpha} \partial_{\alpha}^{B}
$$

acting on elements including first order derivatives and $x_{A B}^{\alpha}$ correspond to derivative coordinates of second order living in $\mathcal{J}^{2}(\mathcal{X})$, the second jet manifold. We will mainly focus on the first order case in the sequel, however the jetstructure and the total derivatives can easily be extended to higher order cases.

We will treat so-called densities in the sequel (a quantity that can be integrated), where we pay special attendance to densities of the form $\mathfrak{F}=\mathcal{F} \mathrm{d} X$ with $\mathcal{F} \in C^{\infty}\left(\mathcal{J}^{1}(\mathcal{X})\right)$ where $\mathrm{d} X$ denotes the volume element on the manifold $\mathcal{D}$, i.e. $\mathrm{d} X=\mathrm{d} X^{1} \wedge \ldots \wedge \mathrm{d} X^{d}$ with $\operatorname{dim}(\mathcal{D})=d$. Since $\mathcal{F} \in C^{\infty}\left(\mathcal{J}^{1}(\mathcal{X})\right)$ is met in our case we restrict ourselves to first order theory and additionally we denote by $F=\int_{\mathcal{D}} \mathfrak{F}$ the integrated quantity, where of course the map $x=\Phi(X)$ leading to $x_{A}=\partial_{A} \Phi(X)$ has to be plugged in to be able to evaluate the integral properly.

Based on the bundle structure $\mathcal{X} \rightarrow \mathcal{D}$ one can introduce several tangent structures, where for our considerations the vertical tangent bundle $\mathcal{V}(\mathcal{X})$ and the cotangent bundles representing differential forms will be used. Of special importance will be the space $\Lambda_{1}^{d}(\mathcal{X})$ induced by the bundle structure $\mathcal{X} \rightarrow \mathcal{D}$

$$
\Lambda_{1}^{d}(\mathcal{X})=\mathcal{T}^{*}(\mathcal{X}) \wedge\left(\stackrel{d}{\Lambda} \mathcal{T}^{*}(\mathcal{D})\right)
$$

with a typical element $\omega=\omega_{\alpha} \mathrm{d} x^{\alpha} \wedge \mathrm{d} X$. It is worth stressing that the functions $\omega_{\alpha}$ may depend on derivative coordinates, however as stated above to simplify the notation no pull backs will be indicated in the forthcoming, i.e. it should be clear from the context which order of derivative is included in the differential form ${ }^{1}$. An important object is the horizontal exterior derivative $\mathrm{d}_{h}$, which meets $\mathrm{d}_{h}(\phi)=\mathrm{d} X^{A} \wedge d_{A}(\phi)$ acting on a differential form $\phi$, where $d_{A}(\phi)$ denotes the Lie-derivative of $\phi$ with respect to $d_{A}$ (see the appendix for more details concerning the relationship of $\mathrm{d}$ and $\mathrm{d}_{h}$ and Stokes theorem in that context).

Furthermore we will treat linear differential operators (of order $k$ ) that are of the following form

$$
\mathfrak{D}: \Lambda_{1}^{d}(\mathcal{X}) \rightarrow \mathcal{V}(\mathcal{X})
$$

that maps an element $\Lambda_{1}^{d}(\mathcal{X})$ of jet-order $p$ to an element $\mathcal{V}(\mathcal{X})$ of jet-order $p+k$. In coordinates we have

$$
\mathfrak{D}(\omega)=\mathfrak{D}^{\alpha \beta \mathfrak{K}} d_{\mathfrak{K}}\left(\omega_{\alpha}\right) \partial_{\beta}, \quad d_{\mathfrak{K}}=d_{A_{k}} \circ \ldots \circ d_{A_{1}} .
$$

with $\omega \in \Lambda_{1}^{d}(\mathcal{X})$. The adjoint operator $\mathfrak{D}^{*}$ follows by integration by parts and fulfills the condition

$$
\left.\mathfrak{D}(\omega)\rfloor \varpi=\mathfrak{D}^{*}(\varpi)\right\rfloor \omega+\mathrm{d}_{h}(\mathfrak{d})
$$

with $\omega, \varpi \in \Lambda_{1}^{d}(\mathcal{X})$, where $\mathfrak{d}$ is a bilinear expression involving the total derivatives up to order $k-1$, see Olver (1986).

\section{SYSTEM REPRESENTATION}

In this section we will introduce port-Hamiltonian systems described by pdes based on a power balance relation. This means that the system is introduced in such a way that the power balance relation together with the structure of the equations represent the physical process. We will consider two cases: (i) the Hamiltonian depends on derivative coordinates, but the operators $\mathcal{J}, \mathcal{R}$ and $\mathcal{G}$ (namely the interconnection, the dissipation and the input/output maps) are just linear maps and (ii) where we relax the assumption regarding $\mathcal{J}, \mathcal{R}$ and $\mathcal{G}$ and consider adequate differential operators $\mathfrak{J}, \mathfrak{R}$ and $\mathfrak{G}$ instead. Before we are able to introduce the corresponding system representations we need to introduce some geometric concepts which will be exploited later on with respect of the derivation of the power balance relation.

Let us consider a vector field which is used to measure the change of the density $\mathcal{F}$. We use a (generalized) vertical vector field $v: \mathcal{X} \rightarrow \mathcal{V}(\mathcal{X})$ locally given as $v=v^{\alpha} \partial_{\alpha}$ where $v^{\alpha}$ may depend on derivative coordinates, together with its first jet-prolongation $j^{1}(v)$ which reads as

$$
j^{1}(v)=v^{\alpha} \partial_{\alpha}+d_{A}\left(v^{\alpha}\right) \partial_{\alpha}^{A} .
$$

Then we compute the Lie-derivative of the densitiy $\mathfrak{F}$ as it has been defined before with respect to the vector field $j^{1}(v)$ and we obtain the important relation

$$
\begin{aligned}
j^{1}(v)(\mathcal{F} \mathrm{d} X) & =\left(v^{\alpha}\left(\partial_{\alpha} \mathcal{F}-d_{A} \partial_{\alpha}^{A} \mathcal{F}\right)+d_{A}\left(v^{\alpha} \partial_{\alpha}^{A} \mathcal{F}\right)\right) \mathrm{d} X \\
& =\left(v^{\alpha} \delta_{\alpha} \mathcal{F}+d_{A}\left(v^{\alpha} \partial_{\alpha}^{A} \mathcal{F}\right)\right) \mathrm{d} X .
\end{aligned}
$$

1 To be more precise: If $\omega_{\alpha} \in \mathcal{J}^{1}(\mathcal{X})$ then $\omega \in\left(\pi_{0}^{1}\right)^{*} \Lambda_{1}^{d}(\mathcal{X})$ with $\pi_{0}^{1}: \mathcal{J}^{1}(\mathcal{X}) \rightarrow \mathcal{X}$. For simplicity we write $\omega \in \Lambda_{1}^{d}(\mathcal{X})$ and suppress the pull back. 
Here the Euler Lagrange operator $\delta$

$$
\delta: \mathcal{J}^{2}(\mathcal{X}) \rightarrow \Lambda_{1}^{d}(\mathcal{X})
$$

comes into play, whose coordinate expressions involves the variational derivative $\delta_{\alpha}$ which acts on $\mathcal{F}$ as

$$
\delta_{\alpha} \mathcal{F}=\partial_{\alpha} \mathcal{F}-d_{A} \partial_{\alpha}^{A} \mathcal{F}
$$

Remark 1. In fact (3) written out in coordinates is a map

with $\mathfrak{F}=\mathcal{F} d X$.

$$
\delta_{\alpha} \mathcal{F} \rightarrow \delta_{\alpha} \mathcal{F} \mathrm{d} x^{\alpha} \wedge \mathrm{d} X
$$

Applying the Theorem of Stokes (see again the appendix) to $(2)$ we find that

$$
\begin{aligned}
\int_{\mathcal{D}} j^{1}(v)(\mathcal{F} \mathrm{d} X) & =\int_{\mathcal{D}} v^{\alpha}\left(\delta_{\alpha} \mathcal{F}\right) \mathrm{d} X+\int_{\partial \mathcal{D}} v^{\alpha}\left(\partial_{\alpha}^{A} \mathcal{F}\right) \mathrm{d} X_{A} \\
& \left.\left.=\int_{\mathcal{D}} v\right\rfloor \delta \mathfrak{F}+\int_{\partial \mathcal{D}} v\right\rfloor \delta^{\partial} \mathfrak{F}
\end{aligned}
$$

with $\left.\mathrm{d} X_{A}=\partial_{A}\right\rfloor \mathrm{d} X$ and the boundary operator

$$
\delta^{\partial} \mathfrak{F}=\partial_{\alpha}^{A} \mathcal{F} \mathrm{d} x^{\alpha} \wedge \mathrm{d} X_{A} .
$$

The relation (4) will be of key interest in the forthcoming, since it provides a natural decomposition of the expression $\int_{\mathcal{D}} j^{1}(v)(\mathcal{F} \mathrm{d} X)$ into a term on the domain $\mathcal{D}$ and one the boundary $\partial \mathcal{D}$.

Important is furthermore the case when the generalized vector-field $v$ is linked to the solution of a pde system (via its semi-group, that $v$ may generate), then the formal change of $F=\int_{\mathcal{D}} \mathcal{F} \mathrm{d} X$ along solutions of a pde system can be computed as $\int_{\mathcal{D}} j^{1}(v)(\mathcal{F} \mathrm{d} X)$ (provided all operations are admissible), which will be denoted by

$$
\dot{F}=\int_{\mathcal{D}} j^{1}(v)(\mathcal{F} \mathrm{d} X)
$$

in this special case.

\subsection{The non-differential Operator case:}

We introduce a port controlled Hamiltonian system on a bundle $\mathcal{X} \rightarrow \mathcal{D}$ in the non-differential operator case in the form of

$$
\begin{aligned}
\dot{x} & =(\mathcal{J}-\mathcal{R})(\delta \mathfrak{H})+u\rfloor \mathcal{G} \\
y & \left.=\mathcal{G}^{*}\right\rfloor \delta \mathfrak{H} .
\end{aligned}
$$

together with appropriate boundary conditions and with a first order Hamiltonian $\mathfrak{H}=\mathcal{H} \mathrm{d} X$ with $\mathcal{H} \in C^{\infty}\left(\mathcal{J}^{1}(\mathcal{X})\right)$. (Here it is worth mentioning that we restrict ourselves to the first order case).

Remark 2. It is worth stressing again, that of course $\delta$ is a differential operator, and the phrase 'non-differential operator case' only refers to the maps $\mathcal{J}, \mathcal{R}$ and $\mathcal{G}$.

We make use of the linear maps (over vector bundles)

$$
\mathcal{J}, \mathcal{R}: \Lambda_{1}^{d} \rightarrow \mathcal{V}(\mathcal{X})
$$

where $\mathcal{J}$ is skew-symmetric and meets

$$
\mathcal{J}(\omega)\rfloor(\varpi)+\mathcal{J}(\varpi)\rfloor(\omega)=0
$$

and since $\mathcal{R}$ is symmetric and positive semi-definite we have

$$
\mathcal{R}(\omega)\rfloor(\varpi)-\mathcal{R}(\varpi)\rfloor(\omega)=0, \quad \mathcal{R}(\omega)\rfloor(\omega) \geq 0
$$

with $\omega, \varpi \in \Lambda_{1}^{d}$.
The input and the output bundles are given as $\mathcal{U} \rightarrow \mathcal{X}$ and $\mathcal{Y} \rightarrow \mathcal{X}$ and the map $\mathcal{G}$ and its adjoint $\mathcal{G}^{*}$ are of the form

with

$$
\mathcal{G}: \mathcal{U} \rightarrow \mathcal{V}(\mathcal{X}), \quad \mathcal{G}^{*}: \Lambda_{1}^{d} \rightarrow \mathcal{Y}
$$

$$
\left.(u\rfloor \mathcal{G})\rfloor \omega=u\rfloor\left(\mathcal{G}^{*}\right\rfloor \omega\right)
$$

Replacing $\mathfrak{F}$ by $\mathfrak{H}$ in (4) and setting $v=\dot{x}$ we obtain

$$
\begin{aligned}
\dot{H} & \left.\left.\left.\left.=\int_{\mathcal{D}}(\mathcal{J}-\mathcal{R})(\delta \mathfrak{H})\right\rfloor \delta \mathfrak{H}+\int_{\mathcal{D}}(u\rfloor \mathcal{G}\right)\right\rfloor \delta \mathfrak{H}+\int_{\partial \mathcal{D}} \dot{x}\right\rfloor \delta^{\partial} \mathfrak{H} \\
& \left.\left.\left.=-\int_{\mathcal{D}} \mathcal{R}(\delta \mathfrak{H})\right\rfloor \delta \mathfrak{H}+\int_{\mathcal{D}} u\right\rfloor y+\int_{\partial \mathcal{D}} \dot{x}\right\rfloor \delta^{\partial} \mathfrak{H}
\end{aligned}
$$

which reflects the power balance, since the total change of the functional $H$ along solutions of (5), is made up of dissipation, collocation on the domain and an expression corresponding to a boundary port (if it exists) depending on the boundary conditions, see Schöberl et al. (2008); Siuka (2011).

Remark 3. Since $\left.\int_{\mathcal{D}} u\right\rfloor y$ in coordinates reads as $\int_{\mathcal{D}} u^{i} y_{i} \mathrm{~d} X$ we interpret the outputs as density valued such that the fibers of the vector bundle $\mathcal{U} \rightarrow \mathcal{X}$ possess the base $e_{i}$ and the fibers of the vector bundle $\mathcal{Y} \rightarrow \mathcal{X}$ possess by duality the base $e^{i} \otimes \mathrm{d} X$. Similarly, this is reflected also in the case of the pairing of $\mathcal{V}(\mathcal{X})$ with $\Lambda_{1}^{d}$ which also takes its values in the densities since with $v \in \mathcal{V}(\mathcal{X})$ and $\omega \in \Lambda_{1}^{d}$ we have $v\rfloor \omega=v^{\alpha} \omega_{\alpha} \mathrm{d} X$.

Casimir functionals Let us consider how to obtain socalled Casimir densities (or functionals) where we restrict ourselves to the first order case only, i.e. $\mathfrak{C}=\mathcal{C} \mathrm{d} X$ with $\mathcal{C} \in C^{\infty}\left(\mathcal{J}^{1}(\mathcal{X})\right)$. Using (4) where we replace $\mathcal{F}$ by $\mathcal{C}$, setting $v=\dot{x}$ and $C=\int_{\mathcal{D}} \mathfrak{C}$ the relation

$$
\left.\left.\left.\left.\dot{C}=\int_{\mathcal{D}}(\mathcal{J}-\mathcal{R})(\delta \mathfrak{H})\right\rfloor \delta \mathfrak{C}+\int_{\mathcal{D}}(u\rfloor \mathcal{G}\right)\right\rfloor \delta \mathfrak{C}+\int_{\partial \mathcal{D}} \dot{x}\right\rfloor \delta^{\partial} \mathfrak{C}
$$

is obtained and a Casimir functional meets

$$
\left.\left.\dot{C}=\int_{\mathcal{D}}(u\rfloor \mathcal{G}\right)\right\rfloor \delta \mathfrak{C} .
$$

This leads to the following two conditions for the Casimir density

$$
\begin{array}{r}
\delta_{\alpha} \mathcal{C}\left(\mathcal{J}^{\alpha \beta}-\mathcal{R}^{\alpha \beta}\right)=0 \\
\left.\left(\dot{x}^{\alpha} \partial_{\alpha}^{A} \mathcal{C}\right)\right|_{\partial \mathcal{D}}=0
\end{array}
$$

which have to be fulfilled (independently of the Hamiltonian density $\mathfrak{H}$ ). If in addition

$$
\left.\left.\int_{\mathcal{D}}(u\rfloor \mathcal{G}\right)\right\rfloor \delta \mathfrak{C}=0
$$

is met, then the density is a conserved quantity.

The concept of control by interconnection based on Casimir functionals using the presented system representation has been applied to a heavy chain system in Schöberl and Siuka (2011) and to the Timoshenko beam in Siuka et al. (2011). The generation of Casimir functionals based on the Stokes-Dirac structure and its consequences for control have been treated for instance in Macchelli et al. (2004b); Pasumarthy and van der Schaft (2007). 


\subsection{The differential operator case:}

A port-Hamiltonian system with respect to the differential operator case reads as

$$
\begin{aligned}
\dot{x} & =(\mathfrak{J}-\mathfrak{R})(\delta \mathfrak{H})+\mathfrak{G}(u) \\
y & =\mathfrak{G}^{*}(\delta \mathfrak{H})
\end{aligned}
$$

together with appropriate boundary conditions, see also Siuka (2011).

For this case the operators $\mathfrak{J}, \mathfrak{R}: \Lambda_{1}^{d} \rightarrow \mathcal{V}(\mathcal{X})$ are $r$-order linear vector valued differential operators where $\mathfrak{J}$ is a skew-adjoint operator according to

$$
\mathfrak{J}(\omega)\rfloor \varpi+\mathfrak{J}(\varpi)\rfloor \omega=\mathrm{d}_{h}(\mathfrak{j})
$$

with

$$
\mathfrak{j}=\mathfrak{j}^{A} \mathrm{~d} X_{A}, \quad \omega, \varpi \in \Lambda_{1}^{d},
$$

cf. (1). Furthermore, $\mathfrak{R}$ is a non-negative self-adjoint operator, i.e.,

$$
\left.\mathfrak{R}(\omega)\rfloor \varpi-\mathfrak{R}(\varpi)\rfloor \omega=\mathrm{d}_{h}(\mathfrak{r}), \quad \mathfrak{R}(\omega)\right\rfloor \omega \geq 0 .
$$

with $\mathfrak{r}=\mathfrak{r}^{A} \mathrm{~d} X_{A}$.

The input operator $\mathfrak{G}$ and its adjoint operator $\mathfrak{G}^{*}$ are maps according to

$$
\mathfrak{G}: \mathcal{U} \rightarrow \mathcal{V}(\mathcal{X}), \quad \mathfrak{G}^{*}: \Lambda_{1}^{d} \rightarrow \mathcal{Y}
$$

and they are linear $r$-order differential operators with respect to the relation

$$
\mathfrak{G}(u)\rfloor \omega=u\rfloor \mathfrak{G}^{*}(\omega)+\mathrm{d}_{h}(\mathfrak{g}), \quad \mathfrak{g}=\mathfrak{g}^{A} \mathrm{~d} X_{A},
$$

Consequently, from (16) we are able to derive the relation

$$
\begin{aligned}
\mathfrak{G}(u)\rfloor \delta \mathfrak{H} & =u\rfloor \mathfrak{G}^{*}(\delta \mathfrak{H})+\mathrm{d}_{h}(\mathfrak{g}) \\
& =u\rfloor y+\mathrm{d}_{h}(\mathfrak{g})
\end{aligned}
$$

where the pairing $u\rfloor y$ will correspond to the domain port. Remark 4. It should be noted that the relations (13), (14) and (16) are the generalizations of (6), (7) and (8) to the differential operator case, where especially the horizontal derivative will lead to appropriate boundary terms when the adjoint operator is taken into account.

The formal change of the Hamiltonian functional $H$ along (12) now takes the form

$$
\left.\left.\left.\dot{H}=\int_{\mathcal{D}}(\mathfrak{J}-\mathfrak{R})(\delta \mathfrak{H})\right\rfloor \delta \mathfrak{H}+\int_{\mathcal{D}} \mathfrak{G}(u)\right\rfloor \delta \mathfrak{H}+\int_{\partial \mathcal{D}} \dot{x}\right\rfloor \delta^{\partial} \mathfrak{H}
$$

and due to the involved differential operators it cannot be concluded in a unique fashion how the power is extracted over the domain and/or the boundary. It is easily checked for instance that plugging in (17) into (18) leads to collocation on the domain and an additional boundary expression. These non-uniqueness depends on the properties of the used differential operators in $\mathfrak{J}, \mathfrak{R}$ and $\mathfrak{G}$ which also has an impact if Casimir functionals are to be considered in this differential-operator case. To be able to draw some conclusions also in that case we will restrict ourselves to specific operators described in the following section:

Specific Operators Motivated by the forthcoming applications we will consider two types of operators.

We introduce a second-order non-negative self-adjoint operator $\mathfrak{R}$ locally given as

$$
\mathfrak{R}(\omega)=d_{A}\left(\mathfrak{R}^{\alpha \beta A B} d_{B}\left(\omega_{\beta}\right)\right) \partial_{\alpha},
$$

with

$$
\mathfrak{R}^{\alpha \beta A B}=\mathfrak{R}^{\beta \alpha B A}, \quad \mathfrak{R}^{\alpha \beta A B} \in C^{\infty}(\mathcal{X}) .
$$

It satisfies the relation

$$
\left.\left.\mathfrak{r}^{A} \mathrm{~d} X_{A}=\bar{\Re}(\omega)\right\rfloor \varpi_{\partial}-\bar{\Re}(\varpi)\right\rfloor \omega_{\partial},
$$

cf. (14) with

$$
\left.\left.\omega_{\partial}=-\partial_{A}\right\rfloor \omega, \quad \varpi_{\partial}=-\partial_{A}\right\rfloor \varpi
$$

where we used

$$
\overline{\mathfrak{R}}(\omega)=\mathfrak{R}^{\alpha \beta A B} d_{B}\left(\omega_{\beta}\right) \partial_{\alpha} .
$$

Remark 5. The condition $\mathfrak{R}^{\alpha \beta A B} \in C^{\infty}(\mathcal{X})$ might be relaxed, and $C^{\infty}(\mathcal{X})$ can be replaced by an appropriate jet-space. Since, in our concrete application this is not the case, we will not focus on that fact more detailed.

The operator $\mathfrak{R}$ is non-negative since $\mathfrak{K}(\omega)\rfloor \omega$ can be written as

$$
-d_{A}\left(\omega_{\alpha}\right) \mathfrak{R}^{\alpha \beta A B} d_{B}\left(\omega_{\beta}\right) \mathrm{d} X+\mathrm{d}_{h}\left(\mathfrak{B}_{\mathfrak{R}}\right)
$$

with the boundary expression

$$
\mathfrak{B}_{\mathfrak{R}}=\omega_{\alpha} \mathfrak{R}^{\alpha \beta A B} d_{B}\left(\omega_{\beta}\right) \mathrm{d} X_{A} .
$$

The non-negativity of the operator follows if

$$
-d_{A}\left(\omega_{\alpha}\right) \mathfrak{R}^{\alpha \beta A B} d_{B}\left(\omega_{\beta}\right) \geq 0
$$

is met.

Furthermore, we define a first-order input operator $\mathfrak{G}$ with

$$
\mathfrak{G}(u)=\mathfrak{G}_{i}^{\alpha A} d_{A}\left(u^{i}\right) \partial_{\alpha}
$$

and, $\mathfrak{G}_{i}^{\alpha A} \in C^{\infty}\left(\mathcal{J}^{1}(\mathcal{X})\right)$ which meets

$$
\left.\mathfrak{G}(u)\rfloor \omega=u\rfloor \mathfrak{G}^{*}(\omega)+\mathrm{d}_{h}(\overline{\mathfrak{G}}(u)\rfloor \omega_{\partial}\right)
$$

with

$$
\left.\overline{\mathfrak{G}}(u)=\mathfrak{G}_{i}^{\alpha A} u^{i} \partial_{\alpha}, \quad \omega_{\partial}=-\partial_{A}\right\rfloor \omega .
$$

The relation (24) takes the form of

$$
\omega_{\alpha} \mathfrak{G}_{i}^{\alpha A} d_{A}\left(u^{i}\right) \mathrm{d} X=-u^{i} d_{A}\left(\omega_{\alpha} \mathfrak{G}_{i}^{\alpha A}\right) \mathrm{d} X+\mathrm{d}_{h}\left(\mathfrak{B}_{\mathfrak{G}}\right)(25)
$$

with

$$
\mathfrak{B}_{\mathfrak{G}}=\omega_{\alpha} \mathfrak{G}_{i}^{\alpha A} u^{i} \mathrm{~d} X_{A} .
$$

Remark 6. As above the condition $\mathfrak{G}_{i}^{\alpha A} \in \mathcal{J}^{1}(\mathcal{X})$ can be relaxed in the same spirit as for the dissipation operator.

\section{EXAMPLES}

In this section we will discuss the presented ideas based on physical examples. We will present the vibrating string example in two fashions -with and without structural damping which affects the $\mathfrak{R}$ operator. To show how an input operator influences the energy flows we analyze a simplified version of magnetohydrodynamics, similar as we did in Schöberl et al. (2010).

\subsection{Vibrating string}

Let us consider the system of a vibrating string. As independent coordinate we choose the spatial coordinate $X$, the dependent coordinates are the deflection $w$ and the temporal momentum $p$. This leads to the following bundle $\mathcal{X} \rightarrow \mathcal{D},(X, w, p) \rightarrow X$, The first jet manifold 
$\mathcal{J}^{1}(\mathcal{X})$ additionally includes the derivative coordinates $w_{X}$ and $p_{X}$ and the boundary $\partial \mathcal{D}$ consists of two points only, namely $X=0$ and $X=L$ where $L$ is the length of the string. Approximately, the pdes take the form

$$
\begin{aligned}
\dot{w} & =\frac{p}{\rho} \\
\dot{p} & =d_{X}\left(P(X) w_{X}\right)
\end{aligned}
$$

with additional boundary conditions and the force in the string is denoted by $P(X)$. To rewrite this system in a Hamiltonian fashion we consider the Hamiltonian density $\mathfrak{H}=\mathcal{H} \mathrm{d} X$ with

$$
\mathcal{H}=\frac{1}{2 \rho} p^{2}+\frac{1}{2} P(X) w_{X}^{2} .
$$

The total energy can be evaluated by $H=\int_{0}^{L} \mathcal{H} \mathrm{d} X$. To obtain partial differential equations in the form as in (5) we can set $\mathcal{R}$ and $\mathcal{G}$ to zero (no damping and no inputs acting on the domain). Then we easily obtain

$$
\left[\begin{array}{c}
\dot{w} \\
\dot{p}
\end{array}\right]=\left[\begin{array}{cc}
0 & 1 \\
-1 & 0
\end{array}\right]\left[\begin{array}{c}
\delta_{w} \mathcal{H} \\
\delta_{p} \mathcal{H}
\end{array}\right]=\mathcal{J}(\delta \mathfrak{H})
$$

with $x=(w, p)$ together with the expression

$$
\delta_{w} \mathcal{H}=\left(\partial_{w}-d_{X} \partial_{w}^{X}\right) \mathcal{H}=-d_{X}\left(P(X) w_{X}\right)
$$

and

$$
\delta_{p} \mathcal{H}=\left(\partial_{p}-d_{X} \partial_{p}^{X}\right) \mathcal{H}=\frac{p}{\rho} .
$$

Evaluating (9) gives

$$
\left.\dot{H}=\int_{\partial \mathcal{D}} \dot{x}\right\rfloor \delta^{\partial} \mathfrak{H}=\left.\dot{w} P(X) w_{X}\right|_{0} ^{L}
$$

and again the power balance relation where the boundary conditions are crucial in the determination of that expression.

Now we extend the equations by structural damping $r>0$ as

$$
\begin{aligned}
\dot{w} & =\frac{p}{\rho} \\
\dot{p} & =d_{X}\left(P(X) w_{X}\right)+d_{X}\left(r d_{X} \frac{p}{\rho}\right) .
\end{aligned}
$$

The port-Hamiltonian representation can be given as

$$
\left[\begin{array}{c}
\dot{w} \\
\dot{p}
\end{array}\right]=\left(\left[\begin{array}{cc}
0 & 1 \\
-1 & 0
\end{array}\right]-\left[\begin{array}{cc}
0 & 0 \\
0 & -d_{X}\left(r d_{X}(\cdot)\right)
\end{array}\right]\right)\left[\begin{array}{c}
\delta_{w} \mathcal{H} \\
\delta_{p} \mathcal{H}
\end{array}\right]
$$

where now a differential operator is utilized in the $\mathfrak{R}$ mapping, as introduced in (19). The power balance reads in this case as

$$
\left.\left.\dot{H}=-\int_{\mathcal{D}} \mathfrak{R}(\delta \mathfrak{H})\right\rfloor \delta \mathfrak{H}+\int_{\partial \mathcal{D}} \dot{x}\right\rfloor \delta^{\partial} \mathfrak{H}
$$

Using (21) we obtain

$$
\left.-\int_{\mathcal{D}} \mathfrak{R}(\delta \mathfrak{H})\right\rfloor \delta \mathfrak{H}=\int_{\mathcal{D}} \dot{w} d_{X}\left(r d_{X}(\dot{w})\right) \mathrm{dX}
$$

where the right hand side of (29) decomposes into

$$
-\int_{\mathcal{D}} d_{X}(\dot{w}) r d_{X}(\dot{w}) \mathrm{d} X+\int_{\partial \mathcal{D}} \dot{w} r d_{X}(\dot{w}) \mathrm{d} X_{A}
$$

and thus

$$
\dot{H}=-\int_{\mathcal{D}} d_{X}(\dot{w}) r d_{X}(\dot{w}) \mathrm{dX}+\int_{\partial \mathcal{D}} \mathfrak{B}_{S}
$$

with the boundary expression

$$
\mathfrak{B}_{S}=\dot{w}\left(r d_{X}(\dot{w})+P(X) w_{X}\right) \mathrm{d} X_{A} .
$$

It is obvious that the structural damping extracts power as can be seen from the term on the domain

$$
-\int_{\mathcal{D}} d_{X}(\dot{w}) r d_{X}(\dot{w}) \mathrm{dX}=-r \int_{\mathcal{D}}\left(d_{X}(\dot{w})\right)^{2} \mathrm{dX}
$$

in $\dot{H}$ since $r>0$.

\subsection{Magnetohydrodynamics (MHD)}

In this section we consider an example that enables us to introduce a differential operator in the in/output mapping, namely so-called inductionless MHD. Roughly speaking, we analyze the macroscopic behavior of an electrically conducting fluid in the presence of external electromagnetic fields. We assume that the dynamic of the additionally induced electromagnetic parts can be neglected (low magnetic Reynold's number). Furthermore, we confine ourselves to the case of negligible viscosity and electrical conductivity.

To obtain the mathematical model we consider again a bundle structure of the form $\mathcal{X} \rightarrow \mathcal{D},\left(X^{A}, q^{\alpha}\right) \rightarrow\left(X^{A}\right)$ and we introduce the so-called material mass and charge density, $\rho(X)$ and $\mu(X)$. Furthermore we assume the existence of a stored energy function $E_{s t}\left(q_{A}^{\alpha}\right)$ which meets

$$
\mathcal{P}=-\rho \frac{\partial E_{s t}}{\partial J}, \quad J=\operatorname{det}(F)
$$

with the deformation gradient $F_{A}^{\alpha}=q_{A}^{\alpha}$, and $F_{A}^{\alpha} \hat{F}_{\alpha}^{B}=\delta_{A}^{B}$, where we have introduced the material pressure $\mathcal{P}(X)$. The electrostatic potential is denoted by $A_{0}(q)$ and the vector potential by $A_{\alpha}(q)$, where the components of the electric field strength are given by $E_{0 \alpha}=\partial_{\alpha} A_{0}$ and of the magnetic flux density by

$$
B_{\alpha \beta}=\partial_{\alpha} A_{\beta}-\partial_{\beta} A_{\alpha} .
$$

The Hamiltonian density $\mathfrak{H}=\mathcal{H} \mathrm{d} X$ follows as

$$
\mathcal{H}=\frac{1}{2 \rho} \delta^{\alpha \beta}\left(p_{\alpha}-\mu A_{\alpha}\right)\left(p_{\beta}-\mu A_{\beta}\right)+\rho E_{s t}
$$

with the momenta

$$
p_{\alpha}=\rho \delta_{\alpha \beta} \dot{q}^{\beta}+\mu A_{\alpha} .
$$

Remark \%. The Hamiltonian can be derived using the Lagrangian

$$
\mathcal{L}=\frac{1}{2} \rho \dot{q}^{\alpha} \delta_{\alpha \beta} \dot{q}^{\beta}-\rho E_{s t}+\mu\left(A_{0}+\dot{q}^{\alpha} A_{\alpha}\right)
$$

together with $\mathcal{H}=\left(p_{\alpha} \dot{q}^{\alpha}-\mathcal{L}\right)$ where the potential $A_{0}$ is neglected in a first step, since it serves as the input, which is considered separately.

The partial differential equations read as

$$
\dot{p}_{\alpha}=-J \hat{F}_{\alpha}^{A} \partial_{A} \mathcal{P}+\mu \frac{1}{\rho} \delta^{\beta \rho}\left(p_{\rho}-\mu A_{\rho}\right) \partial_{\alpha} A_{\beta}
$$

and its Hamiltonian representation follows as

$$
\left[\begin{array}{l}
\dot{q}^{\alpha} \\
\dot{p}_{\alpha}
\end{array}\right]=\left[\begin{array}{cc}
0 & \delta_{\beta}^{\alpha} \\
-\delta_{\alpha}^{\beta} & 0
\end{array}\right]\left[\begin{array}{l}
\delta_{\beta} \mathcal{H} \\
\delta^{\beta} \mathcal{H}
\end{array}\right] .
$$

with $\delta^{\beta} \mathcal{H}=\partial^{\beta} \mathcal{H}=\frac{\partial}{\partial p_{\beta}} \mathcal{H}$. 
Taking the system input $A_{0}$ into account we extend the system and obtain the following representation

$$
\left[\begin{array}{l}
\dot{q}^{\alpha} \\
\dot{p}_{\alpha}
\end{array}\right]=\mathcal{J}\left[\begin{array}{l}
\delta_{\beta} \mathcal{H} \\
\delta^{\beta} \mathcal{H}
\end{array}\right]+\mathfrak{G}\left(A_{0}\right)
$$

and

$$
\mathfrak{G}\left(A_{0}\right)=\left[\begin{array}{c}
0 \\
\mu \hat{F}_{\alpha}^{B} d_{B} A_{0}
\end{array}\right],
$$

where the input map corresponds to the definition of equation (23) with $u=A_{0}$.

The power balance then again takes the form

$$
\left.\left.\dot{H}=\int_{\mathcal{D}} \mathfrak{G}\left(A_{0}\right)\right\rfloor \delta \mathfrak{H}+\int_{\partial \mathcal{D}} \dot{x}\right\rfloor \delta^{\partial} \mathfrak{H}
$$

where the interesting term corresponds to the pairing on the domain including the input and we obtain

$$
\begin{aligned}
\left.\int_{\mathcal{D}} \mathfrak{G}\left(A_{0}\right)\right\rfloor \delta \mathfrak{H} & =\int_{\mathcal{D}} \mu \hat{F}_{\alpha}^{B}\left(d_{B} A_{0}\right) \dot{q}^{\alpha} \mathrm{d} X \\
& =-\int_{\mathcal{D}} d_{B}\left(\hat{F}_{\alpha}^{B} S^{\alpha}\right) A_{0} \mathrm{~d} X+\int_{\partial \mathcal{D}} \mathfrak{B}_{M}
\end{aligned}
$$

with

$$
\mathfrak{B}_{M}=S^{\alpha} \hat{F}_{\alpha}^{B} A_{0} \mathrm{~d} X_{B},
$$

see also (25). Here the adjoint operator on the domain takes the form $\mathfrak{G}^{*}(\delta \mathfrak{H})=-d_{B}\left(\hat{F}_{\alpha}^{B} S^{\alpha}\right)$.

The expression

$$
S^{\alpha}=\frac{\mu}{\rho} \delta^{\alpha \beta}\left(p_{\beta}-\mu A_{\beta}\right)
$$

is equivalent to the components of the convective current density which is caused by the convective transport of charge.

\section{CONCLUSION}

Based on the relation (4) that describes the power balance relation, we introduced port-Hamiltonian systems modeled by partial differential equations, in such a way that energy conservation, dissipation, collocation and energy ports come apparent. This key idea is very similar of that used in the definition of the Stokes-Dirac structure, however due to a different choice of coordinates (not necessarily energy coordinates) the port-Hamiltonian representations of the two approaches differ significantly. This can be observed by a close inspection of the differential operators that are needed to describe the physical systems properly. Therefore it is of great interest for future investigations two analyze and compare these two promising directions more closely.

\section{APPENDIX}

Les us consider a bundle $\pi: \mathcal{X} \rightarrow \mathcal{D},\left(X^{A}, x^{\alpha}\right) \rightarrow\left(X^{A}\right)$ and its n-th order jet manifold $\mathcal{J}^{n}(\mathcal{X})$ as well as the bundle $\pi_{0}^{n}: \mathcal{J}^{n}(\mathcal{X}) \rightarrow \mathcal{X}$ together with a section $\phi: \mathcal{D} \rightarrow \mathcal{X}$, i.e. $x=\phi(X)$.

The exterior derivative $\mathrm{d}$ is connected to the horizontal derivative $\mathrm{d}_{h}$ through

$$
\left(j^{n+1} \phi\right)^{*}\left(\mathrm{~d}_{h}(\omega)\right)=\mathrm{d}\left(\left(j^{n} \phi\right)^{*}(\omega)\right)
$$

for a form $\omega$ living on $\mathcal{J}^{n}(\mathcal{X})$. Roughly speeking the pull back $\left(j^{n} \phi\right)^{*}(\omega)$ denotes $\omega \circ\left(j^{n} \phi\right)$ where $j^{n} \phi$ corresponds to the n-th order jet-lift of the section $\phi$.

Furthermore we have

$\int_{\mathcal{D}} j^{n+1}(\phi)^{*}\left(\mathrm{~d}_{h} \omega\right)=\int_{\mathcal{D}} \mathrm{d}\left(j^{n}(\phi)^{*}(\omega)\right)=\int_{\partial \mathcal{D}} j^{n}(\phi)^{*}(\omega)$ for $\omega \in\left(\pi_{0}^{n}\right)^{*}\left(\Lambda^{d-1}(\mathcal{X})\right)$, which is nothing else than Stokes' theorem adapted to bundles.

\section{ACKNOWLEDGEMENTS}

Markus Schöberl is an APART fellowship holder of the Austrian Academy of Sciences.

\section{REFERENCES}

Giachetta, G., Mangiarotti, L., and Sardanashvily, G. (1997). New Lagrangian and Hamiltonian Methods in Field Theory. World Scientific, Singapore.

Macchelli, A. and Melchiorri, C. (2004). Modeling and control of the timoshenko beam. the distributed port hamiltonian approach. SIAM J. Control Optim., 43, 743-767.

Macchelli, A., van der Schaft, A.J., and Melchiorri, C. (2004a). Port hamiltonian formulation of infinite dimensional systems: Part i modeling. In Proc. 43rd IEEE Conf. Decision and Control (CDC), 3762-3767.

Macchelli, A., van der Schaft, A.J., and Melchiorri, C. (2004b). Port hamiltonian formulation of infinite dimensional systems: Part ii boundary control by interconnection. In Proc. 43rd IEEE Conf. Decision and Control $(C D C), 3768-3773$.

Maschke, B.M. and van der Schaft, A.J. (2005). Compositional Modelling of Distributed-Parameter Systems, 115-154. Advanced Topics in Control Systems Theory. Springer Lect. Notes in Control and Information Sciences, London.

Olver, P.J. (1986). Applications of Lie Groups to Differential Equations. Springer-Verlag, New York.

Pasumarthy, R. and van der Schaft, A.J. (2007). Achievable casimirs and its implications on control of porthamiltonian systems. International Journal of Control, 80, 1421-1438.

Schlacher, K. (2008). Mathematical modeling for nonlinear control: a hamiltonian approach. Mathematics and Computers in simulation, 97, 829-849.

Schöberl, M., Ennsbrunner, H., and Schlacher, K. (2008). Modelling of piezoelectric structures - a hamiltonian approach. Mathematical and Computer Modelling of Dynamical Systems, 14, 179-193.

Schöberl, M. and Siuka, A. (2011). On casimir functionals for field theories in port-hamiltonian description for control purposes. In Proceedings 50th IEEE Conference on Decision and Control (CDC), 7759-7764.

Schöberl, M., Siuka, A., and Schlacher, K. (2010). Geometric aspects of first order field theories in piezoelectricity and magnetohydrodynamics. In Proceedings, International Conference on Electromagnetics in Advanced Applications, 55-58.

Siuka, A. (2011). Geometry, Modelling and Control of Infinite Dimensional Port-Hamiltonian Systems. pHdthesis, Linz. 
Siuka, A., Schöberl, M., and Schlacher, K. (2011). Porthamiltonian modelling and energy-based control of the timoshenko beam - an approach based on structural invariants. Acta Mechanica, 222, 69-89.

van der Schaft, A.J. and Maschke, B.M. (2002). Hamiltonian formulation of distributed-parameter systems with boundary energy flow. Journal of Geometry and Physics, 42, 166-194. 